\title{
Zukunft
}

Rita Albrecht, Klaus Ceynowa, Andreas Degkwitz, Jiri Kende, Thorsten Koch, Gabriele Meßmer, Uwe Risch, Beate Rusch, Robert Scheuerl, Michael Voß

\section{Cloudbasierte Infrastruktur für Bibliotheksdaten - auf dem Weg zu einer Neuordnung der deutschen Verbundlandschaft}

Zusammenfassung: Im Oktober 2012 hatte die Deutsche Forschungsgemeinschaft (DFG) ein Förderprogramm zur Neuausrichtung überregionaler Informationsservices ausgeschrieben, um eine umfassende Reorganisation bestehender Infrastrukturen anzustoßen, die mit den Empfehlungen des Wissenschaftsrates zur Zukunft der bibliothekarischen Verbundsysteme in Deutschland gefordert wurde. Im Themenfeld „Bibliotheksdateninfrastruktur und Lokale Systeme“ der DFG-Ausschreibung wurde das vom Hessischen Bibliotheksinformationssystem (HeBIS), vom Bibliotheksverbund Bayern (BVB) und vom Kooperativen Bibliotheksverbund Berlin-Brandenburg (KOBV) beantragte Vorhaben „Cloudbasierte Infrastruktur für Bibliotheksdaten“ (CIB) bewilligt. Das Projekt zielt auf die Überführung bibliothekarischer Workflows und Dienste in cloudbasierte Arbeitsumgebungen und die sukzessive Ablösung traditioneller Verbund- und Lokalsysteme durch internationale Systemplattformen. $\mathrm{Zu}$ den Arbeitspaketen des Vorhabens gehört u.a. die Einbindung von Norm- und Fremddatenangeboten und von weiteren Services in diese Plattformen.

Schlüsselwörter: Bibliotheks-Informationssystem; Cloud Computing; Informationsinfrastruktur; Forschungsumgebung; Bibliotheksmanagementsysteme

\section{Cloud Based Infrastructure for Library Data - Heading to a Redesigned Landscape of the German Library Consortia}

\begin{abstract}
In October 2012 the Deutsche Forschungsgemeinschaft (DFG) (German Research Foundation) launched a funding programme for the reorganisation of the information services to encourage the redesign of existing infrastructures, which the Wissenschaftsrat (German Research Council) had called for in its recommendations for the future of library union catalogues in Germany. Under the issue of "Library data infrastructure and local systems" the library consortia of Hesse (HeBIS), Bavaria (BVB), and Berlin-Brandenburg (KOBV) applied successfully for the project "Cloud-based Infrastructure for Library Data” (CIB).
\end{abstract}

The goals of the project aim at the transfer of library workflows and services to cloud environments. Traditional union catalogues and local systems will be replaced stepwise by international service platforms. The work packages of the project also include the integration of German authority data as well as further services into these platforms.

Keywords: Library Information Systems; cloud computing; information infrastructure; research environment; library management systems

Rita Albrecht: r.albrecht@ub.uni-frankfurt.de Dr. Klaus Ceynowa: ceynowa@bsb-muenchen.de Dr.Andreas Degkwitz: andreas.degkwitz@ub.hu-berlin.de Jiri Kende: kende@ub.fu-berlin.de Dr. Thorsten Koch: koch@zib.de Gabriele Meßmer: messmer@bsb-muenchen.de Dr. Uwe Risch: risch@hebis.uni-frankfurt.de Beate Rusch: rusch@zib.de Robert Scheuerl: robert.scheuerl@bsb-muenchen.de Dr.Michael Voß:mvoss@ub.hu-berlin.de

\section{Allgemeine Hintergründe}

Dass Märkte nicht vor Ländergrenzen haltmachen, ist eine seit langem bekannte Erfahrung der Globalisierung. Bibliotheken spüren die Folgen der Globalisierung auf dem Informations- und Medienmarkt sehr unmittelbar, etwa in Verhandlungen mit internationalen Verlagen - vor allem im Zusammenhang mit Datenbankangeboten, E-Books und E-Journals. Die Entwicklung hat über den ohnehin stark internationalisierten Markt der Informations- und Kommunikationstechnik nun auch den Markt für lokale Bibliothekssysteme erreicht. Um im globalen Wettbewerb bestehen zu können, haben sich hier vor allem die beiden folgenden Herstellerstrategien durchgesetzt. Zum einen verfolgen die Firmen eine Internationalisierungsstrategie, indem sie ihre Produktentwicklung wie auch ihr Produktangebot nicht primär an nationalen Märkten orientieren, 
sondern sich an den Märkten weltweit und an deren Kundenpotenzialen ausrichten. Die Folge dieser Ausrichtung ist die Standardisierung der Software-Applikationen nach der „Mehrheit“ der Kundenanforderungen, wie sie von Seiten der Firmen auf den internationalen Märkten ermittelt und ausgewertet wird. Der zweite strategische Ansatz liegt in der konsequenten Ausnutzung der IT-Potenziale. Mit der Entwicklung von Cloudlösungen hat sich für die international agierenden Firmen Ex Libris und OCLC die Möglichkeit eröffnet, ihre standardisierten Funktionspakete auf einer stark konsolidierten und weltweit nur an wenigen Standorten vorgehaltenen Systembasis zur Verfügung zu stellen. Auf diese Weise gestalten sich Entwicklung und Distribution des Produktportfolios für die Anbieter wirtschaftlich und für die Bibliotheken bezahlbar, so dass das damit gewählte Geschäftsmodell gute Marktchancen erwarten lässt. Zugleich wird mit diesem Wettbewerbsansatz zumindest teilweise die Globalisierung des Marktes für Bibliothekssysteme vorangetrieben, da nur mit einem weltweiten Kundenstamm die Entwicklung der neuen Systeme rentabel ist.

Vor dem Hintergrund der damit einhergehenden Monopolisierungstendenzen und der daraus erwachsenden Abhängigkeit von derzeit zwei Anbietern für lokale Bibliothekssysteme werden auf der Kundenseite durchaus nachvollziehbare Bestrebungen ausgelöst, den Wettbewerb mit eigenen Open-Source-basierten Lösungen zu beleben. Diese Intentionen sind möglicherweise deshalb gerade in Deutschland zu beobachten, weil in Anbetracht des hier bestehenden Marktumfangs ein ausreichendes Potenzial für „eigene“ Lösungen vermutet wird - dies nicht zuletzt vor dem Hintergrund, dass allen Unkenrufen zum Trotz die deutschen Sonderwege weiterhin Bestand zu haben scheinen. Und in der Tat: Die Normdaten, die verbundübergreifende Fernleihe, die Zeitschriftendatenbank sowie die weit fortgeschrittene Praxis kooperativer Katalogisierung sind Komponenten einer Dateninfrastruktur, die sich als durchaus erfolgreich in der täglichen Arbeit erweist und mit entsprechenden Anpassungen auch in den neuen Arbeitsumgebungen zur Verfügung stehen soll. Allerdings ist es sehr fraglich, ob es sich unter Berücksichtigung der beschriebenen internationalen Entwicklungen lohnt, die anstehenden Herausforderungen überwiegend selbst auf nationaler Ebene in Angriff zu nehmen und vielleicht sogar den Versuch zu unternehmen, die marktbeherrschenden Anbieter mit neuen Entwicklungs- und Lösungsansätzen $\mathrm{zu}$ überholen. Dass die Realisierung lokaler oder auch regionaler Innovationsvorhaben als Modell- oder Pilotlösungen notwendig und unverzichtbar ist, steht außer Zweifel. Doch dass diese Eigenentwicklungen den Herausforderungen eines auf Dauer angelegten Produktivbetriebs gewachsen sind, hat sich auf nationaler Ebene bisher noch nicht als der Regelfall dargestellt. Vielmehr ist dabei eine Vielzahl kleiner 'Innovationsinseln' entstanden, die oft sehr unabhängig voneinander agieren, so dass die notwendige Etablierung marktreifer Produkte ausbleibt. Aus dem Blickwinkel der Wirtschaftlichkeit betrachtet, wäre für die Umsetzung eigener, nationaler Entwicklungsansätze für eine Bibliotheksdateninfrastruktur das Monopol eines verbindlichen, nationalen Angebots unausweichlich und zwar mit allen Nebenwirkungen und Risiken, die monopolistische Strukturen bergen. Denn anders können auf Dauer angelegte Weiterentwicklungen weder finanziert noch wirtschaftlich betrieben werden, wie die gegenwärtige Praxis des Marktes für Informations- und Kommunikationstechnik, aber auch andere Marktsegmente zeigen.

Mit Blick auf den Markt der lokalen Bibliothekssysteme und auf die regionalen Verbundstrukturen hätte ein nationales Monopol einer Katalogisierungsinfrastruktur den wesentlichen Nachteil, dass es auf dem Gebiet der Datenangebote einerseits mit den weltweit agierenden Firmen Ex Libris und OCLC konkurrierte und andererseits für die systemtechnische Umsetzung eines eigenen Datenangebots weitgehend abhängig von der Weiterentwicklung der cloudbasierten Lösungen dieser beiden Hersteller wäre. Mit anderen Worten: Eine deutsche (nationale) Katalogisierungsplattform ist nicht nur genauso ein Monopol wie jedes andere Monopol; denn es dürften neben dieser einen Plattform keine weiteren Plattformen existieren. Zugleich wären Abdeckung und Vollständigkeit einer deutschen Lösung in hohem Maße abhängig von den Firmen Ex Libris und OCLC, so dass die nationale Lösung weder tatsächlich eigenständig noch wirklich wettbewerbsfähig wäre. Denn es kann nicht davon ausgegangen werden, dass die beiden Firmen zugunsten einer nationalen Lösung auf ihre Katalogisierungsplattformen verzichten oder diese in ein deutsches System integrieren würden.

Angesichts der schon jetzt verfügbaren und sich absehbar weiter ausbreitenden Cloud-Lösungen empfiehlt sich für die Neuausrichtung der bibliothekarischen Dateninfrastruktur, zunächst von der lokalen Ebene auszugehen. Denn der Paradigmenwechsel liegt im Übergang von den bisher lokal installierten Anwendungen hin zu Web-basiert nutzbaren Plattformen, deren Datenangebote einerseits zu erweitern und andererseits zu modifizieren sind. Von daher ist in diesen internationalen Plattformen eine an die in Deutschland bestehende Praxis anknüpfende Dateninfrastruktur zur Verfügung zu stellen, um in den Cloudlösungen mit den für die deutsche Praxis bewährten Fremd- und Normdatenangeboten arbeiten zu können. So geht das Projekt „Cloudbasierte Infrastruktur für Bibliotheksdaten“ 
(CIB) ${ }^{1}$, das von den Verbünden HeBIS (Projektkoordinator), BVB und KOBV beantragt und im Rahmen der DFG-Ausschreibung zur Neuausrichtung überregionaler Informationsservices im Themenfeld 1 - Bibliotheksdateninfrastruktur und Lokale Systeme ${ }^{2}$ - bewilligt wurde, nicht von den bestehenden regionalen Verbunddatenbanken, sondern von den lokalen Bibliotheken aus. Leitende Zielsetzungen sind Anpassung und Einbindung der in Deutschland genutzten Nachweis- und Zugangsstrukturen wie Normdaten (GND, ZDB), Fremddaten (shared cataloguing) und Fernleihe in die internationalen Nachweissysteme cloudbasierter Bibliotheksplattformen sowie weitere Fragestellungen, die sich im Zusammenhang mit cloudbasierten Funktionalitäten wie Datenhoheit, Datenschutz, Fernleihe, Zusatzentwicklungen (Apps) etc. ergeben. Damit wird die Entwicklung einer neuen Infrastruktur für Bibliotheksdaten im Hinblick auf die absehbaren Einsatzszenarien für ALMA und WMS verfolgt, ohne dass darüberhinausgehende Marktentwicklungen außer Acht gelassen werden. Im Zuge der Migration in diese neuen Arbeitsumgebungen sollen die regionalen Verbunddatenbanken schrittweise abgelöst und die Funktionalitäten der lokalen Bibliothekssysteme auf die cloudbasierten Plattformen der international agierenden Hersteller überführt werden, was auf der lokalen Ebene zu deutlicher Rationalisierung führt.

\section{Ziele des CIB-Projekts}

Die fortschreitende Internationalisierung der Wissenschaft, ihre zusehends interdisziplinäre Ausrichtung und die durchgängige Netzbasiertheit nahezu aller Produktions- und Publikationsprozesse in Forschung und Lehre erfordern nachhaltig optimierte Recherche- und Zugangsoptionen für global verfügbare Literatur- und Informationsressourcen. Wesentliche Voraussetzung dafür ist in der deutschen Wissenschaftslandschaft die verstärkte Anpassung und Einbindung bestehender Recherche- und $\mathrm{Zu}$ gangsstrukturen in internationale Nachweissysteme. Das CIB-Projekt greift diese Bedarfssituation auf und hat die Entwicklung einer neuen Infrastruktur für Bibliotheksdaten zum Ziel. CIB zielt auf die Integration der Katalogisierung, der Titel- und Bestandsnachweise sowie der Lokalsystemfunktionalitäten in internationale, webbasierte Bibliothekssystem-Plattformen. Diese Plattformen bilden gemäß dem CIB-Modell künftig die primäre Katalogi-

1 http://www.hebis.de/de/1ueber_uns/projekte/cib/Projektantrag_ CIB.pdf.

2 http://www.dfg.de/download/pdf/foerderung/programme/lis/aus schreibung_ueberregionale_informationsservices_121015.pdf. sierungsumgebung und stellen zugleich Lokalsystemfunktionen und auch Endnutzerdienste in Form einer CloudInfrastruktur zur Verfügung. In der Cloud nutzen die Bibliotheken die gleiche Hardware, die gleichen Anwendungsprogramme und die gleichen Daten, ohne dass Softund Hardware für jede Bibliothek separat administriert werden müssen. Damit greift CIB einen globalen Entwicklungsprozess im Bereich moderner Bibliothekssysteme auf und gestaltet diesen aktiv mit, um sowohl auf lokaler Ebene als auch auf darüberliegenden, aggregierten Ebenen zu deutlich optimierten und zugleich wirtschaftlicheren Dienstleistungen zu kommen. Das beantragte Projekt geht dabei von drei grundsätzlichen Prämissen aus:

Erstens: Die Katalogisierung findet zukünftig nicht mehr in regionalen Verbunddatenbanken statt, sondern in einer international ausgerichteten Umgebung mit internationalem Regelwerk. Unter Berücksichtigung der in deutschen Bibliotheken und Bibliotheksverbünden vorhandenen Systemlandschaft ist hierbei auch langfristig von einer maßgeblichen Rolle der zwei großen Bibliothekssystemprovider OCLC und Ex Libris auszugehen, deren aktuelle Systementwicklungen WorldShare (OCLC) und Alma (Ex Libris) auf jeweils globale, cloudbasierte Daten- und Diensteplattformen setzen. Darüber hinaus sind weitere Entwicklungen, die zurzeit in Deutschland noch keine Marktbedeutung haben (zum Beispiel aus dem Open-SourceUmfeld stammende Plattformen), kontinuierlich $\mathrm{zu}$ beobachten und gegebenenfalls in die Strukturbildung einzubeziehen. Die neuen Plattformen zeichnen sich durch die Integration aller Funktionen (Katalogisierung, Erwerbung, Ausleihe, Lizenzverwaltung, Discovery etc.), das Operieren auf umfassenden, internationalen Datenbeständen (WorldCat bei OCLC, Alma Community Zone bei Ex Libris) und die konsequente Nutzung internationaler Regelwerk- und Formatstandards aus. Die Arbeit in dieser neuen Infrastruktur macht die herkömmlichen regionalen Verbunddatenbanken entbehrlich, sie können und sollen nach einer Übergangsphase abgeschaltet werden.

Zweitens: Die heute unter dem Begriff „Lokalsystem“ subsummierten Funktionen und Services werden ebenfalls weitgehend in cloudbasierte Managementsysteme verlagert. Wesentlich ist dabei die Orientierung an der strategischen Ausrichtung der Bibliotheken, sich als Partner von Forschung, Lehre und Studium neu zu positionieren. In einer aus mehreren Schichten bestehenden Architektur werden standardisierbare Funktionen und Services zukünftig zentral erbracht, wobei den internationalen Plattformen von Ex Libris und OCLC wegen ihrer hohen Verbreitung in deutschen wissenschaftlichen Bibliotheken eine wichtige Rolle zufällt. Sukzessive werden damit die Lokalsysteme im herkömmlichen Sinne von einer vor Ort 
(oder alternativ in Form eines ASP-Services) betriebenen und gepflegten Software-Instanz in eine neue Informationsinfrastruktur überführt, womit auf lokaler Ebene deutliche Effizienzgewinne verbunden sind. Die internationalen Plattformen können zudem zur Entwicklung nutzergetriebener, spezifischer Applikationen eingesetzt werden, für die sowohl WorldShare mit der sogenannten „App Galerie“ wie auch Alma auf der Basis offener Schnittstellen bereits spezielle Programmierumgebungen anbieten. Indem diese Plattformen die Erstellung kundenspezifischer Funktionserweiterungen erlauben und darüber hinaus auch über offene Schnittstellen in lokale Dienste eingebunden werden können, zeichnet sich für das zukünftige Dienstleistungsportfolio von Bibliotheken das vielversprechende Bild einer serviceorientierten „Orchestrierung“ von Diensten ab, mit der der Bedarf an lokalspezifischen Zusatzservices gedeckt werden kann.

Drittens: Ein nationaler Datenpool im Sinne eines „nationalen Nachweises“ kann in Form einer virtuellen Sicht auf den deutschen Teilbestand der internationalen Datenplattformen („deutsches Datenfenster“) und in Form von „physischen“ Ausspeicherungen generiert werden. Der nationale Nachweis bildet die Basis für Dienste, die zumindest noch mittelfristig ausschließlich auf deutschen Datenbeständen operieren, z. B. die Fernleihe. Mit der physischen Ausspeicherung kann die redundante Sicherung nationaler Katalogdaten außerhalb der Anbieter-Clouds gewährleistet werden, sofern dies als notwendig betrachtet wird. Beispielsweise können die Daten so als Linked-OpenData zur Weiterverwendung durch Dritte angeboten werden.

Im Zentrum der Arbeit von CIB steht die Gestaltung des technischen, organisatorischen, finanziellen und rechtlichen Übergangs in die neuen Systemwelten. Unter Berücksichtigung der drei Prämissen sind für die großen Arbeitsfelder „Internationale Katalogisierungsplattform“, „Lokale Funktionalitäten in der Cloud“ und „Nationaler Datenpool" folgende Kernkonzepte leitend:

Internationale Katalogisierungsplattform: Das für CIB leitende Infrastrukturmodell für Bibliotheksdaten sieht vor, die Katalogisierung künftig in internationalen Katalogisierungsumgebungen durchzuführen. Dies wird sieht man auf die deutsche Bibliothekslandschaft - im Regelfall entweder die WorldShare-Umgebung von OCLC (mit dem WorldCat als globalem Datenpool) oder die Alma-Umgebung von Ex Libris (mit der Community Zone als ebenfalls globalem Datenpool) sein. Durch Synchronisation beider Plattformen werden in beiden Systemumgebungen alle deutschen Katalogdaten verfügbar gemacht, was wiederum die Grundlage für entsprechende Sichten und Ausspeicherungen zum Beispiel in Form fachlicher oder thematischer Teilbestände bilden kann. So wird etwa in der Kooperationsvereinbarung des CIB-Konsortiums mit Ex Libris explizit betont: „This cooperation includes the implementation and application of the technical requirements that will enable smooth and adequate collaboration among German libraries within the Alma platform as well as the mutual synchronization of the cataloguing data of German libraries between Alma and the respective platform of OCLC (WMS).“

Der WorldCat enthält bereits jetzt die Daten von HeBIS und BVB, des GBV, des BSZ und der DNB, die Daten der nordrhein-westfälischen Verbundbibliotheken werden aufgrund der dort verfolgten Open-Data-Policy voraussichtlich in absehbaren Fristen integrierbar sein. Somit steht WorldCat bereits zu Projektbeginn als zentrale Fremddatenquelle für deutsche Bibliotheksdaten und zur Erzeugung selektiver Datensichten zur Verfügung. Ein wesentliches Ziel des Projekts ist jedoch, dass alle Daten auf den internationalen Plattformen Alma und WorldCat synchronisiert zur Verfügung stehen und gegebenenfalls in Zukunft auch auf weiteren Plattformen verfügbar sind.

Lokale Funktionalitäten in der Cloud: Die bisherigen „Integrierten Bibliothekssysteme“ werden abgelöst durch Dienste und Services aus cloudbasierten Systemen. Systemumgebungen wie OCLC WorldShare oder Ex Libris Alma werden Exemplar- und Ausleihdaten, Erwerbungsund Haushaltsdaten, Lieferanten- und Benutzerdaten sowie alle dazugehörenden Vorgangs- bzw. Bewegungsdaten und Statusinformationen enthalten. Sie können damit ein großes Spektrum an herkömmlichen Lokalsystemfunktionalitäten (Erwerbung, Ausleihe etc.) künftig zentral bereitstellen. Als global eingesetzte Systeme, die sehr heterogenen Benutzerbedürfnissen genügen müssen, werden sie bereits im Standardfunktionsumfang komfortable Konfigurationsmöglichkeiten bieten.

Da die neuen cloudbasierten lokalen Services weltweit zum Einsatz kommen werden, können sie jedoch auf bestimmte nationale, regionale oder lokale Besonderheiten und Anforderungen nur begrenzt Rücksicht nehmen. Für weitergehende, u. a. auf spezifische lokale Anforderungen reagierende Zusatzapplikationen, stellen beide PlattformAnbieter daher offene Programmierumgebungen zur Verfügung. Damit sind Ergänzungen des Standardfunktionsumfangs oder Abwandlungen vorhandener Funktionen realisierbar. Die Anwender sind nicht mehr ausschließlich darauf angewiesen, Änderungswünsche einzubringen und vom Systemhersteller umsetzen zu lassen, sondern können diese Aufgabe selbst übernehmen oder von dritter Seite ausführen lassen. Dies ermöglicht eine auf das individuelle Profil jeder einzelnen Bibliothek zugeschnittene Erweiterung des Daten- und Diensteangebotes. Mit Ent- 
wicklungsbedarf ist primär im Bereich der Kopplung an Systeme der Universitätsverwaltung $\mathrm{zu}$ rechnen (insbesondere Anbindung von Buchhaltungs-/Haushaltssystemen, von E-Science- und E-Learning-Systemen sowie von Campus-Management-Systemen). Weiterer Handlungsbedarf kann zum Beispiel beim Aufbau und der Bereitstellung lokaler Sammlungen und standortspezifischer Anforderungen entstehen (z.B. Data Mining auf selektierten thematischen Corpora).

Nationaler Datenpool: In der von CIB konzipierten Dateninfrastruktur kann ein „nationaler Datenpool“ als Gesamtheit aller deutschen Katalogdaten direkt aus den internationalen Katalogisierungsplattformen erzeugt werden. Dies kann sowohl in Gestalt eines „Deutschen Datenfensters“, als selektive Sicht auf den nationalen Teildatenbestand, als auch in Form einer physischen OnlineAusspeicherung der nationalen Katalogdaten im Sinne eines echten Datenabzugs erfolgen. Als primäre Ziele einer derartigen Ausspeicherung sind die Erstellung einer Sicherungskopie deutscher Katalogdaten sowie die Aufbereitung des nationalen Datenpools für Linked-Open-Data-Applikationen zu nennen; beides verlangt die Extraktion der deutschen Teilbestände aus den internationalen Systemumgebungen.

$\mathrm{Zu}$ betonen ist, dass im hier skizzierten Modell die nationale Datenplattform nicht als Katalogisierungsumgebung und auch nicht als Endnutzerschnittstelle fungiert, sondern ausschließlich für Dienste genutzt wird, die dezidiert einen auf nationale Datenbestände begrenzten Fokus haben. Der nationale Datenpool ist damit eine Funktion, aber nicht der Kern der vorgeschlagenen Infrastruktur und kann daher auch ohne signifikanten Ressourcenaufwand bereitgestellt werden.

Technisch betrachtet werden die Anbieter der internationalen Plattformen eine nationale Teilmenge der Daten („deutsches Datenfenster“) zur Verfügung stellen, die sofern diese Ausspeicherung als notwendig betrachtet wird - auf einem deutschen, sicherheitszertifizierten Server gespiegelt wird. Diese Teilmenge wird laufend mit den internationalen Plattformen abgeglichen. Sie enthält sämtliche Titel mit vollständigen Metadaten, Anreicherungselementen und Bestandsnachweisen der beteiligten Bibliotheken sowie mindestens die damit verknüpften Normdatensätze als eigene Entitäten. Hierfür sind insbesondere die Update-Mechanismen konkret zu spezifizieren, und es ist zu klären, wer diesen Server betreiben soll. Für letztere Aufgabe kommen drei Modelle in Frage, die hinsichtlich ihrer Wirtschaftlichkeit zu untersuchen sind: Betrieb des „nationalen Servers“ durch einen der Anbieter der internationalen Plattformen selbst; Betrieb durch eine der am CIB-Projekt beteiligten Einrichtungen; Auslagerung an eine „dritte“ Institution, zum Beispiel eine neu zu gründende Betreibergesellschaft.

Mit dem Einstieg in die internationalen Systemumgebungen verbindet sich ein Paradigmenwechsel für die anwendenden Bibliotheken, der nicht nur die System- und Applikationsebene, sondern zukünftig auch Datenhaltung und Datenstrukturen betrifft, die ebenfalls in den cloudbasierten Bibliotheksmanagementsystemen der Systemprovider vorgehalten werden. Insbesondere mit Blick auf die zukünftige zentralisierte Datenhaltung sind belastbare und wirksame Möglichkeiten der Beteiligung und Mitwirkung der Bibliotheken bei der Gestaltung der vorgesehenen Entwicklungsschritte zu finden. Der überregionale, strukturbildende Ansatz des CIB-Vorhabens besteht im konsequenten Umstieg auf eine internationale Katalogisierungsumgebung, mit der das bisherige Regionalprinzip deutscher Verbundarbeit auf diesem Gebiet nicht nur reduziert, sondern vollständig aufgegeben wird. Nationale Sonderwege, insbesondere in der Format- und Regelwerksentwicklung, werden damit ebenso vermieden wie die derzeitige starke Heterogenität auf der Ebene der Lokalsysteme und der regionalen Verbundsysteme. Das CIBModell reagiert damit zugleich auf den dringenden Bedarf der Umstellung von einer primär regional ausgerichteten Dateninfrastruktur hin zu einer internationalen bibliothekarischen Daten- und Diensteumgebung. Damit wird zudem der international arbeitenden Wissenschaft Rechnung getragen, die von einer Informationsinfrastruktur profitieren wird, deren primärer Fokus auf globalen anstelle von nationalen oder gar regional geprägten Informationsangeboten und -diensten liegt.

Der Umstieg der am CIB-Konsortium beteiligten Verbünde in die neuen, internationalen Systemumgebungen soll nach drei Jahren pilotartig und zum Ende der insgesamt fünfjährigen Projektlaufzeit (vorbehaltlich der positiven Evaluierung durch die Deutsche Forschungsgemeinschaft) weitgehend vollständig erfolgt sein. Damit verbindet sich auch die Option, künftig als Servicebroker für interessierte Bibliotheken deutschlandweit aufzutreten. Die Entwicklung und Erprobung entsprechender Geschäftsmodelle, in denen die Verbünde als Vertragspartner gegenüber den Systemprovidern und IT-technischen Betreibern der CloudStrukturen („Regional Nodes“) auftreten und im Gegenzug die entsprechenden Dienste und Applikationen den teilnehmenden Bibliotheken „aus einer Hand“ anbieten können, sind ebenfalls Teil des CIB-Projekts. 


\section{Integration und Synchronisation von Datenbeständen}

Die beiden bereits existierenden Katalogisierungsplattformen sind im Hinblick auf ihre bibliographischen Daten, Norm- und Verlagsdaten etc. weiter zu entwickeln. Der über Jahrzehnte aufgebaute und in der Gemeinsamen Normdatei (GND) zusammengeführte Pool an Normdaten für Personen, Körperschaften, Geographika, Kongresse und Sachschlagworte (sowie Werktitel der Musik) stellt eine wichtige Errungenschaft des deutschen Bibliothekswesens dar. Mit der Deutschen Nationalbibliothek (DNB) sowie den Anbietern der derzeit am Markt existierenden Plattformen (Ex Libris, OCLC) wurde deshalb vereinbart, dass Normdaten für die deutschsprachigen Länder in die internationalen Katalogisierungsplattformen eingebunden werden.

Ziel ist es, die Normdaten unmittelbar beim Katalogisierungsvorgang zur Verfügung zu haben und bei Bedarf auch Änderungen oder Neueintragungen zu ermöglichen ein Wechsel der Arbeitsumgebung zu einem Fremdsystem soll nicht notwendig sein. Die Verknüpfung zwischen einem Titeldatensatz und Normdatensätzen wird datentechnisch als Verlinkung von zwei Datensätzen über eine Identifikationsnummer hergestellt. Die GND bleibt als Normdatenquelle erhalten, jedoch können auch die internationalen Plattformen Normdaten aufnehmen. Über ein Synchronisationsverfahren wird sichergestellt, dass die Normdaten in allen beteiligten Datenquellen entsprechend aktualisiert werden. Dieser Systemaufbau hat den Vorteil, dass auch die Daten der nicht am CIB-Projekt beteiligten Verbünde sowie von Institutionen außerhalb der bibliothekarischen Verbundsysteme einbezogen und genutzt werden können.

Die Zeitschriftendatenbank (ZDB) hat in den deutschsprachigen Ländern bezogen auf die Titelentitäten fortlaufender Sammelwerke ebenfalls den Charakter einer Normdatei. Ihre Integration in den WorldCat ist in Vorbereitung. Was für die Gemeinsame Normdatei gilt, trifft im Rahmen des CIB-Projekts auch für die ZDB zu. So muss auch hier ein komfortabler Onlinezugriff und gegebenenfalls eine Onlinesynchronisation eingerichtet werden. Die ZDB-Nummer als Identifikationsnummer bleibt erhalten.

Im Hinblick auf die Fremddatenübernahme garantieren die internationalen Katalogisierungsplattformen aufgrund der hohen Anzahl vorhandener bibliographischer Daten aus weltweit verteilten Quellen, dass die Quote der Eigenkatalogisate auch für weniger „gängige“ Materialien (z.B. für nicht-lateinische Schriftzeichen) deutlich absinken wird. Im Projektverlauf werden, soweit erforderlich, weitere Fremddatenquellen in die Katalogisierungsplattformen integriert.

Mit dem Umstieg einer Bibliothek von der Verbundkatalogisierungsumgebung in die internationale Katalogisierungsumgebung verändern sich notwendigerweise die Datenflüsse. Zumindest für eine Übergangszeit müssen die Verbunddatenbanken mit den internationalen Katalogisierungsplattformen synchronisiert werden. Auf diesem Weg kann sichergestellt werden, dass von Bibliotheken mit einem „traditionellen Bibliothekssystem“ in eine Verbunddatenbank eingebrachte Daten zeitnah in die internationale Plattform repliziert werden und dort zur Nachnutzung bereit stehen. In gleicher Weise müssen die in eine internationale Plattform eingebrachten Daten einer Verbundbibliothek in die entsprechende Verbunddatenbank zurückfließen, um für all jene Dienste im Verbund zur Verfügung zu stehen, die noch auf die zentrale Verbunddatenbank angewiesen sind.

Für diese Verfahren der Datensynchronisation sind verschiedene technische Lösungen (Batch oder Online) denkbar, die zumindest mit dem OCLC WorldCat schon heute weitgehend realisiert sind. Auch der weiterhin bestehende Bedarf an Datenmigrationen, etwa bei Aufnahme neuer Verbundmitglieder, bei Altdatenmigrationen oder bei der Migration von Sonderkatalogen muss abgedeckt werden. Mit den Herstellern ist ein Verfahren abzustimmen, bei dem die Katalogdaten inklusive Besitznachweise direkt in die internationalen Katalogisierungsumgebungen eingespielt werden. Die damit notwendigerweise verbundene Konversion der Daten in das benötigte Format und der Abgleich mit der zukünftig zu verwendenden Katalogisierungsumgebung können auch weiterhin als Dienstleistung der regional zuständigen Verbundzentrale angeboten werden.

Mit diesen Übergangsszenarien und den damit entwickelten Verfahren und Schnittstellen wird gleichzeitig die Grundlage geschaffen, dass auch Verbünde, die zunächst nicht an dem Projekt CIB beteiligt sind, zu einem späteren Zeitpunkt folgen können. Durch die Nutzung der verfügbaren Schnittstellen ist es problemlos möglich, dass diese - wenn gewünscht - weiterhin eigenständig bleiben und dennoch die internationalen Plattformen eine vollständige Abbildung eines „Nationalen Datenfensters“ enthalten.

Wesentlich für das CIB-Projekt ist die Synchronisierung der internationalen Plattformen untereinander, so dass der Datenbestand der deutschen Bibliotheken als ein einheitliches „Deutsches Datenfenster“ darstellbar und gegebenenfalls in Form einer physikalischen Ausspeicherung als „Nationaler Datenpool“ zur Verfügung steht. Hierauf könnten gegebenenfalls weitere Dienste wie die Fern- 
leihe, Katalogdatenanreicherung, Datenbereinigungen etc. aufgesetzt werden. Die Anbieter Ex Libris und OCLC haben sich in den mit dem CIB-Konsortium abgeschlossenen Kooperationsvereinbarungen bereit erklärt, entsprechende Synchronisierungsverfahren zwischen ihren Plattformen zu entwickeln.

\section{Lokale Bibliothekssysteme in der Cloud}

Das Themenfeld 1 der DFG-Ausschreibung zur Neuausrichtung der Informationsinfrastrukturen hieß bewusst „Bibliotheksdateninfrastruktur und Lokale Systeme“; denn aufgrund der technologischen Entwicklung wird ein großer Teil der Funktionspalette heutiger „integrated library systems“ (Lokalsysteme) zukünftig als lizenzierbarer Dienst statt als (stand alone-)System angeboten. Die cloudbasierten Bibliothekssysteme, die jetzt entwickelt und in ersten Bibliotheken eingesetzt werden, bilden daher konsequenterweise die Basis des CIB-Projekts.

Im Zentrum der Betrachtung stehen die Nachfolgesysteme der in Deutschland am meisten verbreiteten Bibliothekssysteme der Anbieter Ex Libris und OCLC, die beide heute nicht nur als Softwarehersteller, sondern auch als Anbieter von Datendiensten auftreten ${ }^{3}$. Mit dem WorldCatIndex (OCLC) und Primo Central (Ex Libris) sowie mit anderen Produkten, wie den Knowledge Bases zur Verwaltung von lizenzpflichtigen E-Ressourcen, bieten beide Firmen bereits seit einigen Jahren Datendienste an, die mit den eigenen Softwareprodukten am effektivsten genutzt werden können. Die Dienste werden von beiden Anbietern auf cloudbasierten Plattformen - ALMA von Ex Libris und WorldShare Management Services (WMS) von OCLC - gehostet.

Mit der weiteren Entwicklung der cloudbasierten Bibliothekssysteme wird sich perspektivisch auch die Bandbreite realisierbarer Dienste weiter vergrößern und eine flexible Kombinierbarkeit je nach Bedarfslage ermöglichen. Beide Anbieter betonen, dass ihre Plattformen über Web-Services adressierbar sind und dass sie Schnittstellen in Form von Application Programming Interfaces (APIs) bereitstellen, die es einerseits ermöglichen, die Daten-

$3 \mathrm{Zu}$ künftigen Bibliothekssystemen vgl. die Übersicht von KemnerHeek, Kirstin: Konzeption und Angebot zukünftiger Bibliotheksmanagementsysteme: Bestandsaufnahme und Analyse. - Kölner Arbeitspapiere zur Bibliotheks- und Informationswissenschaft, Band 64, 2012. http://www.fbi.fh-koeln.de/institut/papers/kabi/volltexte/ band064.pdf. dienste in anderen als den eigenen Umgebungen zu nutzen und andererseits die eigenen Softwarelösungen durch extern programmierte Funktionen zu erweitern.

Offene Schnittstellen sind eine wichtige Voraussetzung für das Gelingen des CIB-Projekts, sei es für die Synchronisierung der Daten zwischen den beiden im Vordergrund stehenden Plattformen, sei es für die Anbindung externer Dienste, Programme und Anwendungen, oder sei es für die Interaktion mit anderen Plattformen und Systemen. Das CIB-Projekt konzentriert sich derzeit exemplarisch auf die beiden Anbieter Ex Libris und OCLC, da diese absehbar die einzigen sind, die über einsatzfähige Plattformen verfügen. Zur Realisierung der Projektziele wurden inzwischen mit diesen beiden Anbietern Kooperationsvereinbarungen abgeschlossen, wobei das CIB-Konzept erklärtermaßen offen für die Berücksichtigung weiterer kommerzieller oder Open-Source-basierter Lösungen ist.

Der Wechsel zu den cloudbasierten Systemen bedeutet für viele Bibliotheken einen Paradigmenwechsel. Bislang wurden sowohl die Bibliotheksdaten als auch die Bibliothekssoftware entweder auf eigenen Servern der Bibliotheken oder auf zentralen Servern des lokalen Rechenzentrums beziehungsweise vom jeweils regionalen Bibliotheksverbund gehostet und gepflegt. In den cloudbasierten Systemen werden die Daten wie auch die Software auf der Plattform des jeweiligen Anbieters gehalten und von den Firmen selbst betrieben. Zugleich bemühen sich die Firmen, in unterschiedlicher Weise auf regionale Besonderheiten einzugehen. OCLC strebt beispielsweise ein Partnermodell an, bei dem Lokalsystemfunktionalitäten auf „regional nodes“ - z.B. bei Verbünden - gehostet werden können. Bei Alma können in der sogenannten „network zone“ spezifische Datenangebote, die von mehreren, kooperierenden Bibliotheken gemeinsam genutzt werden, aufgebaut und zur Verfügung gestellt werden.

Der Wechsel in die Cloud bringt vielfältige Vorteile, aber auch Herausforderungen mit sich: Für die Auftragsdatenverarbeitung in der Cloud sind der Datenschutz und die Datensicherheit eine besondere Herausforderung. Die Vorteile der neuen Systemarchitektur liegen auf der Hand - Wegfall der Aufwendungen für die laufende Erneuerung der Hardware, für die Updates der Betriebs- und Datenbanksysteme sowie für die Updates der Bibliothekssoftware. Damit wird der Rechner, ebenso wie der Softwarebetrieb, weitgehend von den Systemanbietern verantwortet. Bei der bibliothekarischen Arbeit und bei der Systemadministration werden Effizienzgewinne und Synergieeffekte u.a. durch Reduktion der Vielfalt der zu betreibenden und $\mathrm{zu}$ bedienenden Systeme sowie durch die Zusammenführung der Workflows für Print- und EMedien erzielt. 
Die beiden im Rahmen des Projekts im Fokus stehenden cloudbasierten Bibliothekssysteme Alma und WMS weisen vergleichbare Konzepte auf und verfügen über eine ähnliche serviceorientierte Architektur. Beide Systeme werden nur als „Software as a Service“ (SaaS) auf Basis von Lizenzen und Service Level Agreements angeboten. Die Datenhaltung erfolgt in mandantenfähigen Datenbanken, beide Systeme sind als offene Plattformen konzipiert, die auf der Basis von Standardschnittstellen wie OpenURL, SRU, Z39.50, EDI, SIP2, NCIP etc. und über weitere Webservices die Anbindung von universitären und anderen externen Systemen ermöglichen.

Auf das komplette Funktionsspektrum von ALMA und WMS kann und soll hier nicht im Detail eingegangen werden; deshalb werden nur die wichtigsten Strukturmerkmale genannt. Beide Systeme stellen eine Katalogdatenplattform und eine entsprechende Anwendungssoftware als Funktionskomponenten zur Verfügung. Beide Katalogdatenplattformen verfügen über eine „public cloud“ (Community Zone bei Alma, WorldCat bei OCLC) und über eine „private cloud“, die - in beiden Systemen unterschiedlich gestaltet - den Bibliotheken die Möglichkeit bietet, die Katalogdaten um bibliotheksspezifische Angaben anzureichern, die von ihnen bevorzugten Normdaten zu nutzen und nicht zuletzt besonders sensible Daten geschützt vorzuhalten; Letzteres gilt insbesondere für Erwerbungs- und Ausleihdaten.

Beide Plattformen decken alle grundlegenden Bibliotheksprozesse im Bereich Erwerbung, Katalogisierung und Ausleihe ab. Zudem bieten beide Plattformen die Integration von Tools für Datenanalyse und -auswertung und eines Linking- und Discovery-Systems an. Um darüber hinaus ein hohes Maß an Flexibilität zu erreichen, werden von beiden Plattformen Wege zu einer Funktionserweiterung der Software eröffnet: OCLC bietet über eine „App Gallery“ die Möglichkeit, „Apps“ zur Funktionserweiterung von WMS einzusetzen, seien diese nun selbst entwickelt oder von Dritten zugekauft. Bei Alma werden anwenderseitig entwickelte Zusatzprogramme über die offene Plattform „EL Commons“ bzw. direkt in Alma zur Verfügung gestellt.

Die Analyse der vorhandenen Systemfunktionalitäten, insbesondere im Hinblick auf deutsche Besonderheiten und deren (Weiter-)Entwicklung in Kooperation mit den Anbietern - wie z.B. die Einbindung der GND oder der ZDB - sind grundlegende Arbeitspakete des CIB-Projekts, um im Ergebnis Dateninfrastrukturen zur Verfügung zu stellen, die für die deutsche Katalogisierungspraxis weiterhin eingesetzt werden sollen. Beide Anbieter garantieren den Bibliotheken die Datenhoheit und freie Verfügbarkeit über ihre Daten und machen keine Rechte an den Daten der Bibliotheken geltend. Die Daten europäischer Kunden werden von beiden Anbietern in Eigenverantwortung in Rechenzentren innerhalb der Europäischen Union gehalten, die den europäischen Normen für Datenschutz und Datensicherheit unterliegen. Die konkrete Prüfung der Anforderungen an Datenschutz und Datensicherheit der cloudbasierten Systeme ist ebenfalls Bestandteil des CIBProjekts. Die Endnutzeranwendungen der Bibliotheken oder Verbünde wie z.B. Primo oder VuFind sind nicht Bestandteil des CIB-Projekts. Welche Endnutzeranwendungen zum Einsatz kommen, wird nicht vorgegeben und ist auch hinsichtlich der genutzten Daten beziehungsweise Indices flexibel.

\section{Zusammenfassung und Ausblick}

Mit dem Einstieg in diese Infrastruktur verbinden sich für die teilnehmenden Bibliotheken und Verbünde als Mehrwerte signifikante Effizienzsteigerungen. $\mathrm{Zu}$ nennen sind unter anderem der Verzicht auf Hardwarebeschaffungen und -pflege vor Ort, deutlich verringerter Aufwand für die Systemadministration sowie eine weitaus effizientere Anwendungsbetreuung durch die Zentralisierung von Softwareupdates, Versionswechseln und Fehlerbehebungen. Wenn sich die Bibliothek vor Ort nicht mehr um Hardware, Software, Systemupdates und -pflege kümmern muss, stehen freiwerdende Ressourcen für Innovationsaufgaben und unmittelbar endnutzerbezogene Services zur Verfügung. Die zu erwartenden Effizienzgewinne bei der Abwicklung von Routineaufgaben ermöglichen zugleich deutliche Verbesserungen des Recherche- und Zugangsspektrums und der kooperativen Nutzung von Datenstrukturen. Für Wissenschaftlerinnen und Wissenschaftler wird damit die Auffindbarkeit der jeweils relevanten Fachinformation signifikant gesteigert und der Zugang dazu erheblich erleichtert. Nicht zuletzt wird mit diesem Ansatz - wie vom Wissenschaftsrat und der Deutschen Forschungsgemeinschaft gefordert - die bisherige Struktur der redundanten regionalen Verbunddatenbanken schrittweise überwunden und die Bibliotheksdateninfrastruktur in Deutschland durch Einbettung in internationale Arbeitsstrukturen in mittelfristiger Perspektive nachhaltig verändert.

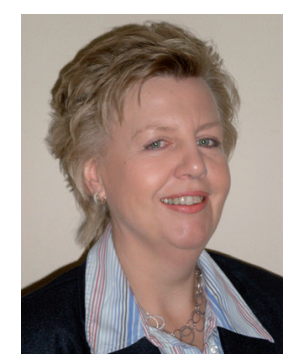

Rita Albrecht

HeBIS-Verbundzentrale

Universitätsbibliothek Johann Christian

Senckenberg

Bockenheimer Landstraße 134-138

D-60325 Frankfurt

r.albrecht@ub.uni-frankfurt.de 


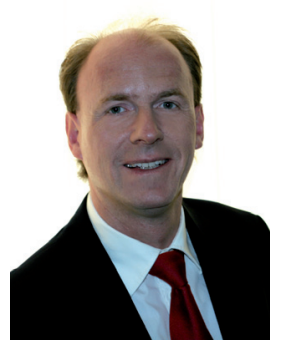

\section{Dr. Klaus Ceynowa}

Stellvertretender Generaldirektor

Bayerische Staatsbibliothek

Ludwigstraße 16

D-80539 München

ceynowa@bsb-muenchen.de

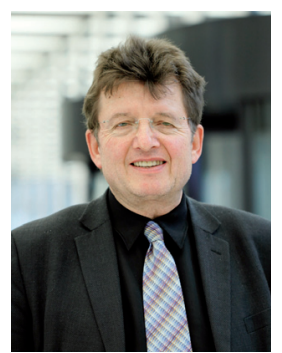

\section{Dr. Andreas Degkwitz}

Direktor der Universitätsbibliothek der Humboldt-Universität zu Berlin Unter den Linden 6 D-10099 Berlin andreas.degkwitz@ub.hu-berlin.de

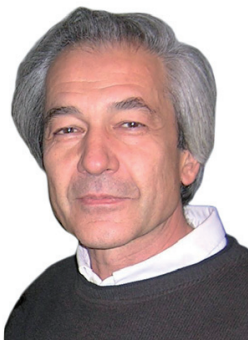

\section{Jiri Kende}

Universitätsbibliothek der FU Berlin Garystraße 39

D-14195 Berlin

kende@ub.fu-berlin.de

\section{Dr. Thorsten Koch}

Kooperativer Bibliotheksverbund Berlin-Brandenburg

Konrad-Zuse-Zentrum für

Informationstechnik Berlin

Takustraße 7

D-14195 Berlin

koch@zib.de

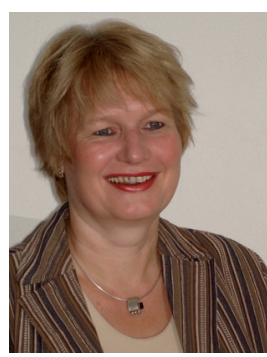

\section{Gabriele Meßmer}

Bayerische Staatsbibliothek

Ludwigstraße 16

D-80539 München

messmer@bsb-muenchen.de

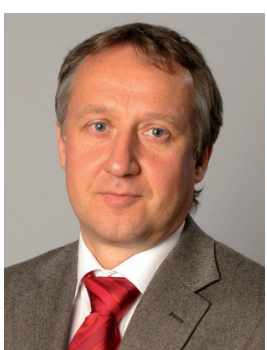

\section{Dr. Uwe Risch}

Leiter HeBIS Verbundzentrale

Universitätsbibliothek Johann Christian

Senckenberg

Bockenheimer Landstraße 134-138

D-60325 Frankfurt am Main

risch@hebis.uni-frankfurt.de

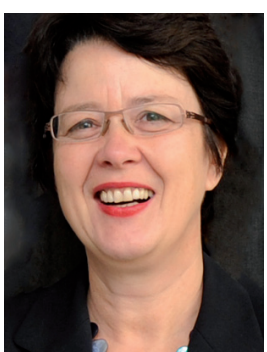

\section{Beate Rusch}

Kooperativer Bibliotheksverbund

Berlin-Brandenburg

Konrad-Zuse-Zentrum für

Informationstechnik Berlin

Takustraße 7

D-14195 Berlin

rusch@zib.de

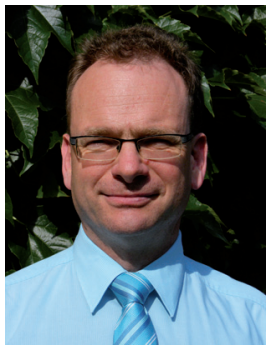

\section{Robert Scheuerl}

Bayerische Staatsbibliothek

Bibliotheks-Verbund Bayern/

Verbundzentrale

Ludwigstraße 16

D-80539 München

robert.scheuerl@bsb-muenchen.de

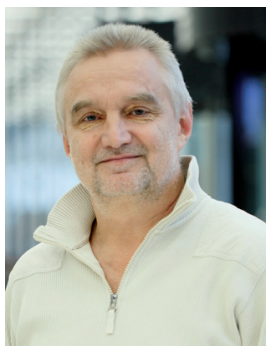

\section{Dr. Michael Voß}

Universitätsbibliothek der Humboldt-Universität zu Berlin

Unter den Linden 6

D-10099 Berlin

mvoss@ub.hu-berlin.de 